\title{
Are homozygous SLC19A3 deletions non-responsive to thiamine/biotin?
}

\author{
Josef Finsterer ${ }^{\circ}$
}

Klinik Landstrasse, Messerli Institute, Austria.

With interest, we read the article by Değerliyurt et al. ${ }^{1}$ about a 5yo male with biotin-thiamine-responsive Leigh syndrome due to a homozygous deletion in SLC19A3. Despite treatment with thiamine and biotin and a triple anti-seizure drug (ASD) therapy the patient presented clinically with severe developmental delay, intractable seizures, intermittent dystonia, axial hypotonia, and quadruspasticity and became progressive. ${ }^{1}$ The cerebral MRI showed bilaterally symmetric T2/ FLAIR hyperintense lesions in the basal ganglia (initially sparing the caudate nucleus), the pons, and bilaterally symmetric DWI hyperintensities in the peri-rolandic areas. ${ }^{1}$ We have the following comments and concerns.

The main shortcoming is that genetic studies had been carried out only in the index patient and not in other affected or non-affected firstdegree relatives. Since the deletion occurred in the homozygous form and one sibling had died from Leigh syndrome at age $2 y$, it is quite likely that either parent each carried the variant in the heterozygous form. In this respect, we should know if any of the parent's relatives were clinically affected by a neurodegenerative or multisystem disease.

A second shortcoming is that it remains unexplained why the patient was progressive despite receiving an appropriate treatment. We should know if the patient truly adhered to the therapy or if thiamine and biotin were not regularly taken after some time as indicated in the report. ${ }^{1}$ It is also conceivable that the patient

\section{Josef Finsterer}

fifigs1@yahoo.de

Received 24th January 2020, accepted 16th July 2020. adhered to the treatment but that the condition was non-responsive to treatment. It is also conceivable that the dosage was too low or that there was an interference between the vitamins and other drugs (e.g. ASDs) ${ }^{2}$ which prevented adequate resorption of the vitamins or induced accelerated elimination after resorption.

A third shortcoming is that the patient's triple antiepileptic medication was not mentioned. Initially, he received phenobarbital (PB) and phenytoin (PHT). From both these ASDs it is well-known that they are mitochondriontoxic. ${ }^{3}$ We should know if these ASDs were discontinued and replaced during the course or if the patient was still taking them at the last follow-up. It is conceivable that mitochondriontoxicity of ASDs contributed to the progression of the phenotype and the involvement of the caudate nucleus on follow-up MRIs despite initial improvement of the imaging findings.

A fourth shortcoming is that no discussion was hold about the possibility that all the lesions seen on MRI were multi-locular stroke-like lesions (SLLs). ${ }^{4}$ Since at least the supra-tentorial lesion were hyperintense on DWI and also the infra-tentorial lesions were described as DWI hyperintense, we should know if corresponding ADC maps were hyperintense, isointense, or hypointense. Knowing if a cytotoxic or vasogenic edema was present is crucial as therapy may be different from that applied in this case. If the lesions were interpreted as SLLs, NO-precursors could have been beneficial. ${ }^{5}$ If it is was a cytotoxic edema, cardiac embolism, thus involvement of the heart in the metabolic disorder, needs to be excluded.

Overall, this interesting case has a number of shortcomings which need to be solved 
before final conclusions can be drawn. Drugadherence, drug toxicity, nature of imaging abnormalities, and causes of progression need to be extensively discussed. It is also crucial for genetic counselling of the family that the genetic status of other first degree relatives, in particular the parents, is known.

Key words: SLC19A3, mitochondrial, thiamin, mtDNA, mitochondrial, biotin.

\section{REFERENCES}

1. Değerliyurt A, Gündüz M, Ceylaner S, Ünal Ö, Ünal S. Neonatal form of biotin-thiamine-responsive basal ganglia disease. Clues to diagnosis. Turk J Pediatr 2019; 61: 261-266.
2. Rodriguez JJ, Acosta F, Bourgeois L, Dasgupta A. Biotin at high concentration interferes with the LOCI digoxin assay but the PETINIA phenytoin assay is not affected. Ann Clin Lab Sci 2018; 48: 164-167.

3. Finsterer J. Toxicity of antiepileptic drugs to mitochondria. Handb Exp Pharmacol 2017; 240: 473488.

4. Bhatia KD, Krishnan P, Kortman H, Klostranec J, Krings T. Acute cortical lesions in MELAS syndrome: anatomic distribution, symmetry, and evolution. AJNR Am J Neuroradiol 2020; 41: 167-173.

5. Ganetzky RD, Falk MJ. 8-year retrospective analysis of intravenous arginine therapy for acute metabolic strokes in pediatric mitochondrial disease. Mol Genet Metab 2018; 123: 301-308.

\title{
Response to "Neonatal form of biotin-thiamine-responsive basal ganglia disease. Clues to diagnosis"
}

\author{
Aydan Değerliyurt ${ }^{1 \oplus}$, Serdar Ceylaner ${ }^{2 \oplus}$ \\ Department of Pediatric Neurology, Ankara Children's Hematology-Oncology Training and Research Hospital, Ankara; ${ }^{2}$ Intergen \\ Genetics Centre, Ankara, Turkey.
}

Thank you for your interest in our report ${ }^{1}$ and the commentary you provided.

If we start with genetics, this case has a homozygote whole gene deletion. In such a gross deletion case, family screening for probable heterozygote cases is frequently done using the MLPA method. MLPA commercial kits are produced by a single company all over the world and there is no kit for the SLC19A3 gene. PCR gel electrophoresis method can be used for family screening but it cannot make differential diagnosis between heterozygote deletions and those that are normal. Gap-PCR testing is not useful for whole gene deletions as detection of breakpoints is very difficult. Whole genome sequencing or Q-PCR may be used for this study but these applications are very expensive. In such homozygote cases when parents are relatives, the possibility of UPD or de novo deletions are "extremely low" and are mostly not present in daily routine. On the other hand, gross deletion is a very well-known mechanism for this disorder and there is no confusion concerning if this is a disease causing variant or not. As there was no confusion, healthy sibling screening was not a deficiency in showing if this deletion was disease causing for this patient or not. In summary, family screening is conducted in all cases with single gene disorders in our daily routine but in this case, due to the type of mutation and limitations of techniques worldwide, we did not screen this family. 
Biotin-thiamine-responsive basal ganglia disease (BTBGD) is a progressive disorder that can result in severe sequela and death if not treated. Such poor prognosis is especially common in patients with early neonatal Leighlike syndrome and the early infantile-onset form of BTBGD, as in our patient, even when treated with biotin and thiamine. ${ }^{2,3}$ The fact that these patients show neurodevelopmental problems in various degrees despite early treatment $t^{4,5}$ indicates that it may be too late to prevent permanent brain damage even when treatment is started at the time of the first symptoms. ${ }^{6}$

As we also mentioned in the article, our patient had not regularly used the vitamin treatment started during the neonatal period until he was diagnosed when 3 years old. The disease had therefore progressed until the time of diagnosis and the patient had developed dystonia in addition to motor retardation. He used the treatment regularly once the definite diagnosis was made and the symptoms did not worsen afterwards. However, he did not fully respond to the treatment and the sequela remained as the basal ganglia damage was already irreversible. The lack of response to treatment following the development of permanent damage in the cerebral areas as a result of a long untreated period has also been demonstrated previously for this disorder. ${ }^{7}$

Despite the lack of a general consensus, the recommended doses in BTBGD treatment are $10-40 \mathrm{mg} / \mathrm{kg} / \mathrm{d}$ for thiamine (with increased doses suggested during the acute crisis period) and $5-10 \mathrm{mg} / \mathrm{kg} / \mathrm{d}$ for biotin. ${ }^{2,8}$ The doses of 30 $\mathrm{mg} / \mathrm{kg} / \mathrm{d}$ thiamine and $10 \mathrm{mg} / \mathrm{kg} / \mathrm{d}$ biotin started after our patient was 3 years old are within the recommended ranges in the literature. Our patient is now 7 years old and the clinical picture is stable. Head control is present but he still needs support to sit and can only say a few words. The intermittent dystonia that appears with stimulus and the infrequent seizures continue. He is being treated for the seizures with topiramate, clobazam and levetiracetam, which have been cleared for use in BTBGD. ${ }^{2}$
Our patient has been undergoing cardiac investigations including echocardiography since the neonatal period for the metabolic disorder and there is no cardiac pathology.

Classic BTBGD presents with typical MRI findings demonstrating dominant involvement of the bilateral caudate heads and the putamen, as originally reported by Ozand et al. ${ }^{9}$ There may be various degrees of cortex, subcortical white matter, thalamus, cerebellum and brainstem involvement in addition to significant symmetrical edema of the bilateral caudate heads and the putamen in the acute stage. $^{10,11}$ Once the acute stage is over, the abnormal signal changes at the caudate head and the putamen are usually permanent. It is not possible for a cerebrovascular accident or a stroke to cause bilateral and symmetrical involvement of so many different and extensive areas. Our patient had no cardiac involvement that could cause an embolus. Signal changes together with significant edema in the thalamus and putamen, bilateral cortical involvement, and brainstem involvement were present at the time of first presentation in the newborn period, as seen in almost all BTBGD patients with the acute neonatal form of the disorder. There was no caudate involvement in the MRI at the time of the first presentation. What we wanted to emphasize in this paper was the absence of caudate involvement, although involvement of the putamen, cortex, thalamus, and brainstem was present bilaterally, in the acute stage in patients with neonatal type BTBGD in contrast to the classic form. Although this has been mentioned in some previous articles, it has not been emphasized as a characteristic of the neonatal form. ${ }^{12,13}$ We would like to emphasize once again that basal ganglia disease responsive to biotin and thiamine treatment, one of the treatable types of Leigh syndrome, should be considered and treatment started immediately if the MRI shows no caudate involvement despite cortex, putamen, thalamus, and brainstem involvement bilaterally on MRI in a newborn patient with encephalopathy and lactic acidosis. 
In closing, we would also like to pay our respects to the eminent Turkish pediatrician Dr. Pinar Ozand who was the first to introduce this metabolic disorder, for which recognition is of great significance as it is treatable, into the medical agenda with the original article he wrote following his careful observations.

\section{REFERENCES}

1. Değerliyurt A, Gündüz M, Ceylaner S, Ünal Ö, Ünal S. Neonatal form of biotin-thiamine-responsive basal ganglia disease. Clues to diagnosis. Turk J Pediatr 2019; 61: 261-266.

2. Tabarki B, Al-Hashem A, Alfadhel M. Biotinthiamine-responsive basal ganglia disease. In: Adam $\mathrm{MP}$, Ardinger $\mathrm{HH}$, Pagon RA, Wallace SE, Bean LJH, Stephens K, Amemiya A, (eds). GeneReviews ${ }^{\circledR}$ [Internet]. Seattle (WA): University of Washington, Seattle; 1993-2020.

3. Algahtani H, Ghamdi S, Shirah B, Alharbi B, Algahtani R, Bazaid A. Biotin-thiamine-responsive basal ganglia disease: catastrophic consequences of delay in diagnosis and treatment. Neurol Res 2017; 39: 117-125.

4. Savasta S, Bassanese F, Buschini C, et al. Biotinthiamine responsive encephalopathy: report of an Egyptian family with a novel SLC19A3 mutation and review of the literature. J Pediatr Genet 2019; 8: 100-108.

5. Kamaşak T, Havalı C, İnce H, et al. Are diagnostic magnetic resonance patterns life-saving in children with biotin-thiamine-responsive basal ganglia disease? Eur J Paediatr Neurol 2018; 22: 1139-1149.
6. van der Knaap MS, Kevelam SH. Reply: infantile leigh-like syndrome caused by SLC19A3 mutations is a treatable disease. Brain 2014; 137(Pt 9): e296.

7. Flønes I, Sztromwasser P, Haugarvoll K, et al. Novel SLC19A3 promoter deletion and allelic silencing in biotin-thiamine-responsive basal ganglia encephalopathy. PLoS One 2016; 11: e0149055.

8. Tabarki B, Alfadhel M, AlShahwan S, Hundallah K, AlShafi S, AlHashem A. Treatment of biotinresponsive basal ganglia disease: open comparative study between the combination of biotin plus thiamine versus thiamine alone. Eur J Paediatr Neurol 2015; 19: 547-552.

9. Ozand PT, Gascon GG, Al Essa M, et al. Biotinresponsive basal ganglia disease: a novel entity. Brain 1998; 121(Pt 7): 1267-1279.

10. Kassem H, Wafaie A, Alsuhibani S, Farid T. Biotinresponsive basal ganglia disease: neuroimaging features before and after treatment. AJNR Am J Neuroradiol 2014; 35: 1990-1995.

11. Alfadhel M, Almuntashri M, Jadah RH, et al. Biotinresponsive basal ganglia disease should be renamed biotin-thiamine-responsive basal ganglia disease: a retrospective review of the clinical, radiological and molecular findings of 18 new cases. Orphanet J Rare Dis 2013; 8: 83 .

12. Gerards M, Kamps R, van Oevelen J, et al. Exome sequencing reveals a novel Moroccan founder mutation in SLC19A3 as a new cause of earlychildhood fatal Leigh syndrome. Brain 2013; 136(Pt 3): $882-890$.

13. Ortigoza-Escobar JD, Serrano M, Molero M, et al Thiamine transporter-2 deficiency: outcome and treatment monitoring. Orphanet J Rare Dis 2014; 9: 92. 